\title{
Molecular lifting, twisting, and curling during metal-assisted polycyclic hydrocarbon dehydrogenation.
}

\author{
Davide Curcio ${ }^{1}$, Luca Omiciuolo ${ }^{1}$, Monica Pozzo ${ }^{2}$, Paolo Lacovig $^{3}$, Silvano Lizzit ${ }^{3}$, \\ Naila Jabeen $^{1,4,5}$, Luca Petaccia ${ }^{3}$, Dario Alfè ${ }^{2}$, Alessandro Baraldi ${ }^{* 1,3,6}$ \\ ${ }^{1}$ Physics Department, University of Trieste, Via Valerio 2, 34127 Trieste, Italy \\ ${ }^{2}$ Department of Earth Sciences, Department of Physics and Astronomy, Thomas Young Centre@UCL, London Centre for \\ Nanotechnology, University College London, Gower Street, London WC1E 6BT, United Kingdom \\ ${ }^{3}$ Elettra-Sincrotrone Trieste S.C.p.A., Strada Statale 14 Km 163.5, 34149 Trieste, Italy \\ ${ }^{4}$ International Centre for Theoretical Physics, Strada Costiera 11, 34151 Trieste, Italy \\ ${ }^{5}$ Nanosciences \& Catalysis Division, National Centre for Physics, Islamabad 44000, Pakistan \\ ${ }^{6}$ IOM-CNR, Laboratorio TASC, AREA Science Park, S.S. 14 km 163.5, 34149 Trieste, Italy \\ *Corresponding Author: alessandro.baraldi@elettra.eu
}

\section{Supporting Information}



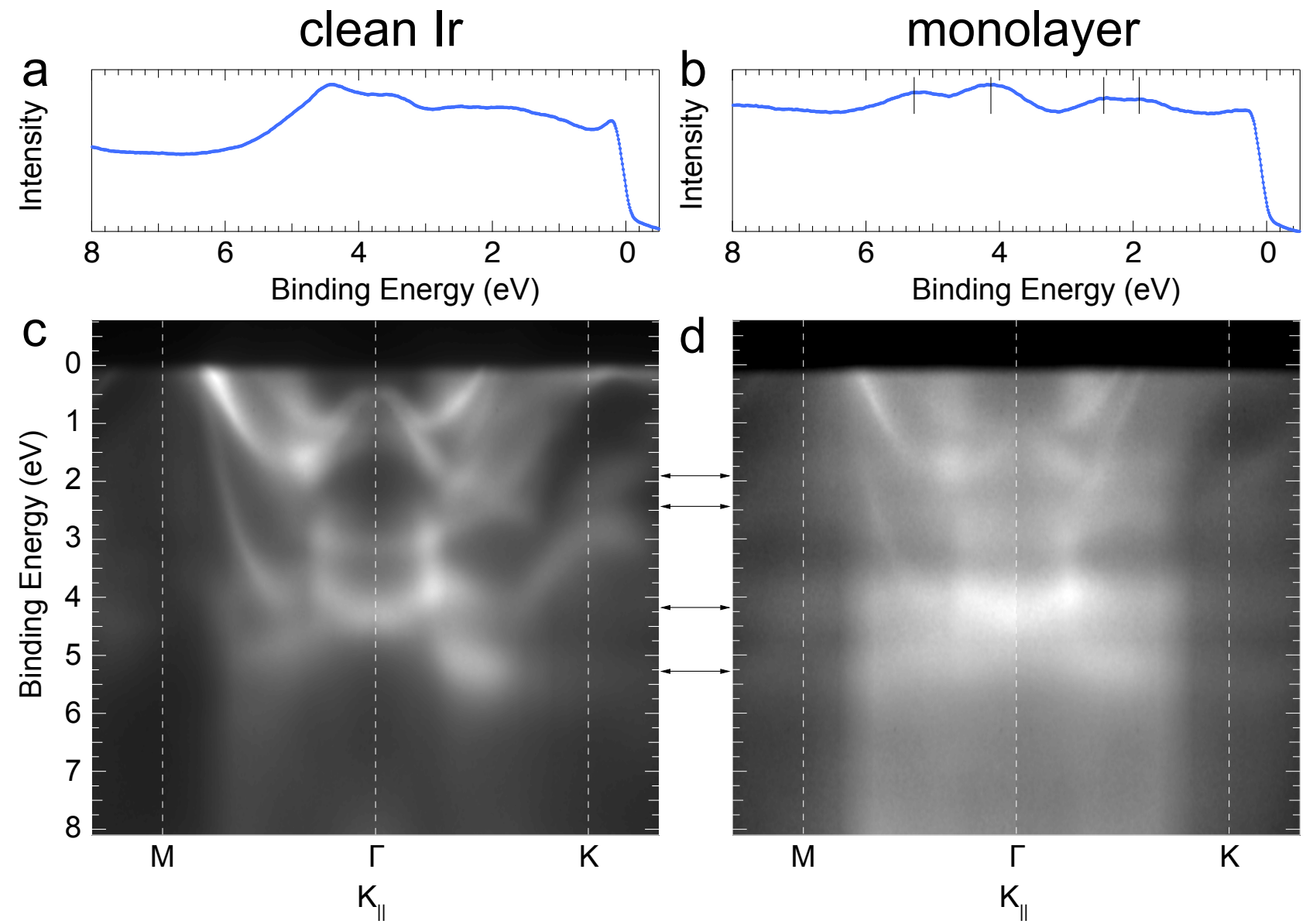

Figure S1. (a) Energy Dispersion Curve (EDC) for the clean $\operatorname{Ir}(111)$ obtained by selecting a vertical cut of the bottom ARPES image at the M point. (b) EDC curve for a monolayer of coronene on $\operatorname{Ir}(111)$ obtained by selecting a vertical cut of the bottom ARPES image at the M point. The molecular levels are shown superimposed (vertical bars). (c) ARPES map along the $\mathrm{M}-\Gamma-\mathrm{K}$ direction for the clean $\operatorname{Ir}(111)$ acquired at $\mathrm{h} v=34 \mathrm{eV}$. (d) ARPES map along the $\mathrm{M}-\Gamma-\mathrm{K}$ direction acquired (hv=34 eV) after deposition of a monolayer of coronene on $\operatorname{Ir}(111)$. The four clearly visible non-dispersing molecular levels are marked with arrows. 


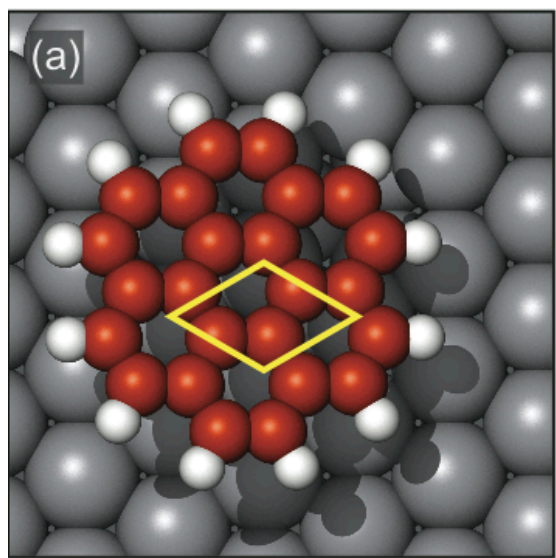

$E=-0.183 \mathrm{eV}$

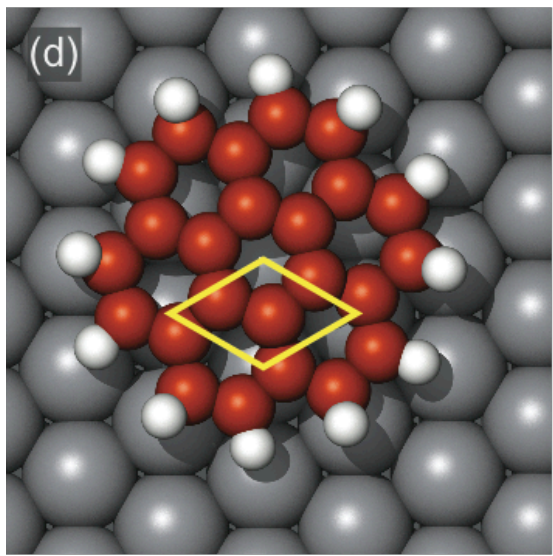

$\mathrm{E}=+1.28 \mathrm{eV}$

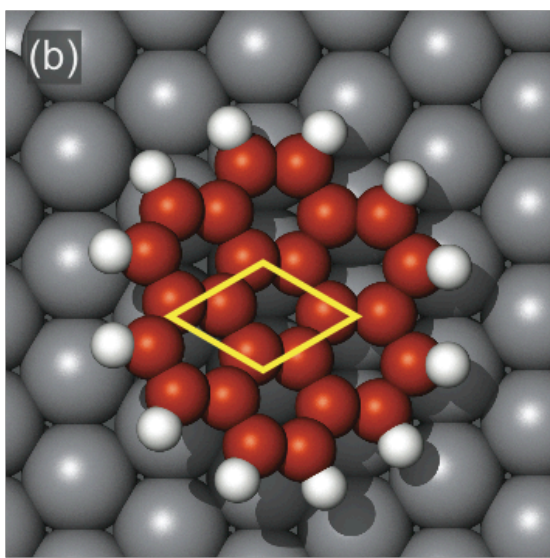

$E=-0.67 \mathrm{eV}$

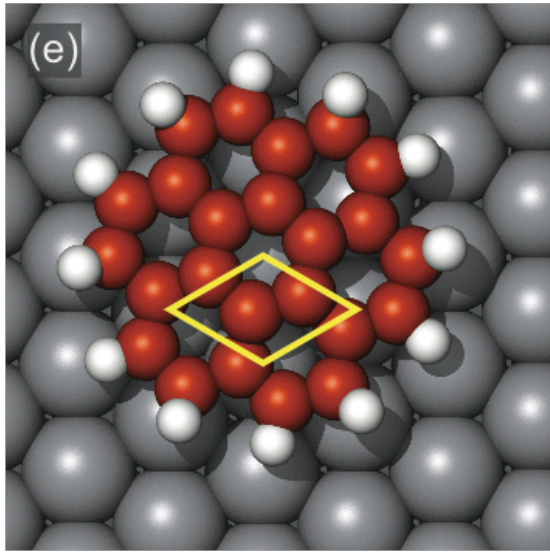

$\mathrm{E}=+1.26 \mathrm{eV}$

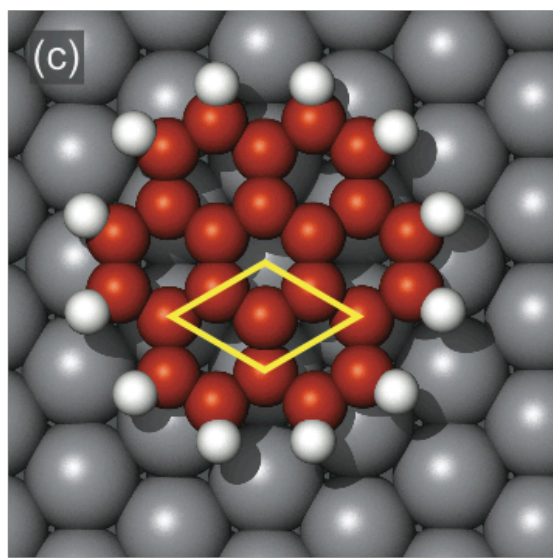

$\mathrm{E}=+0.87 \mathrm{eV}$

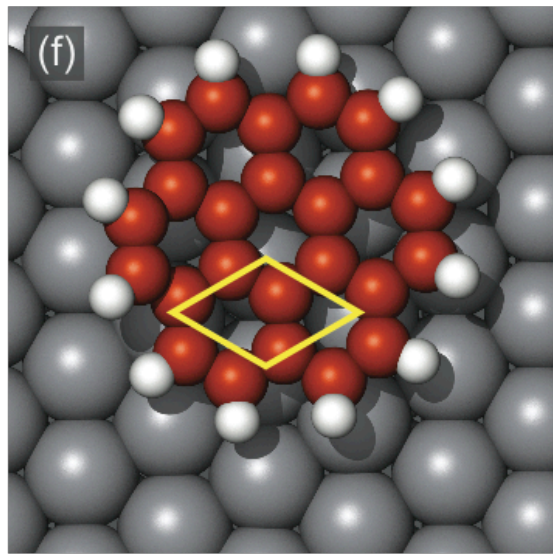

$E=+1.94 \mathrm{eV}$

Figure S2. Additional adsorbed molecular configurations probed by DFT. The yellow parallelogram in each panel represents the substrate elementary cell, with each corner on a top position. The adsorption energies are calculated with respect to the gas phase molecule configuration. The minimum energy configuration, described in the article (see Fig. 2), has an adsorption energy of $-1.46 \mathrm{eV}$. 


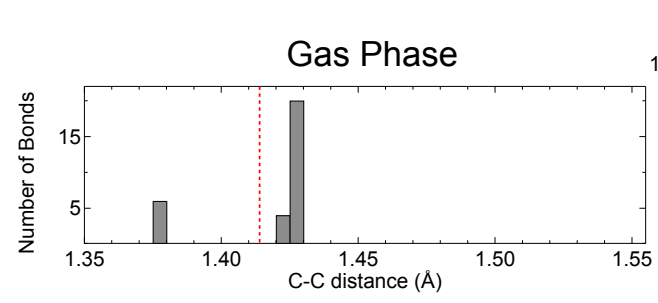

C-C distance $(\AA)$
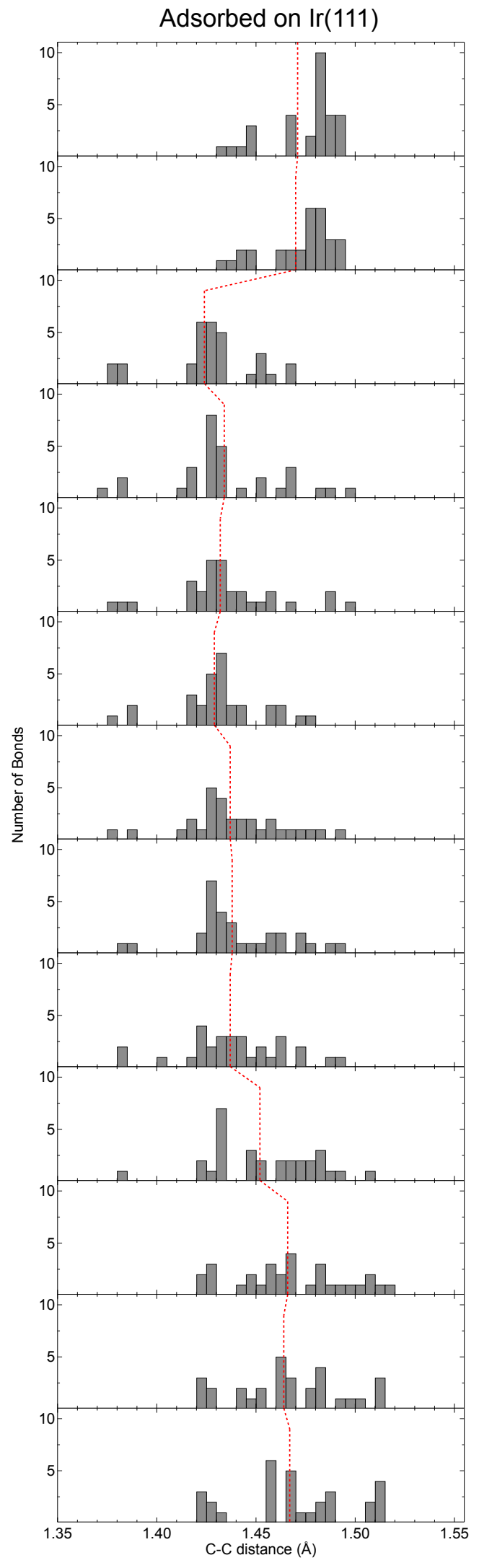

\section{Figure S3.}

(left) $\mathrm{C}-\mathrm{C}$ bond length histograms for gas phase (top), and adsorbed coronene during dehydrogenation, as obtained from DFT calculations. All the $30 \mathrm{C}-\mathrm{C}$ bonds were included. The average $\mathrm{C}-\mathrm{C}$ bond length for each dissociation step is also reported (dashed red line).

(right) Schematic representation of gas phase (top), and adsorbed coronene during dehydrogenation of $\operatorname{Ir}(111)$. The $\mathrm{C}$ atoms are colored according to the mean distance from the 3 (or 2) nearest neighbors. The color scale is reported on top. 


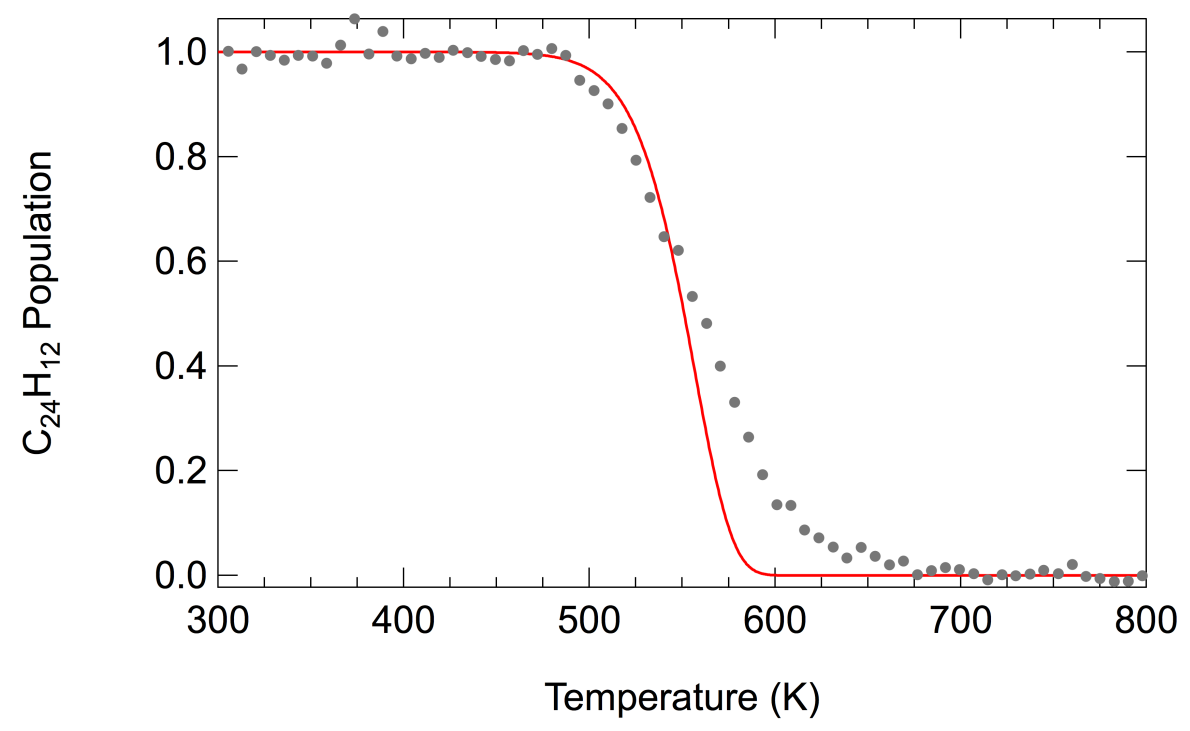

Figure S4. Experimental $\mathrm{C}_{24} \mathrm{H}_{12}$ population (grey dots) as calculated from the experimental data by judging the photoemission intensity evolution around $285 \mathrm{eV}$ BE (main article, Fig. 3), where the main contribution is expected to be due to undissociated coronene. The population is normalized to the initial intensity. The rate equation theoretical model for the first dissociation, obtained from the DFT calculated energy barrier $(1.32 \mathrm{eV})$ and assuming a $10^{10}$ pre-exponential factor, is also shown (red curve). At temperature above $550 \mathrm{~K}$, the observed broadening of the experimental curve is due to the spectral contribution arising from the high $\mathrm{BE}$ tail of the $\mathrm{C}$ 1s signal due to dissociated molecules. 